\title{
On metrizability of a class of 2-manifolds with linear connection
}

\author{
Alena Vanžurová
}




\title{
ON METRIZABILITY OF A CLASS OF 2-MANIFOLDS WITH LINEAR CONNECTION
}

\author{
ALENA VANŽUROVÁ
}

\begin{abstract}
In [17], we proved a theorem which shows how to find, under particular assumptions that guarantee metrizability (among others, recurrency of the curvature is necessary), all (at least local) pseudo-Riemannian metrics compatible with a given torsion-free linear connection without flat points in a domain of two-dimensional manifold. The result has the form of an implication only. In general, if there are flat points, or if curvature is not recurrent, we cannot give any good answer as it can also be demonstrated by examples. Note that in higher dimension, the problem of metrizability is not easy to solve, [6,7]. Here, we try to apply this apparatus to the class of (torsion-free, locally homogeneous) connections with constant Christoffels in open domains of 2-manifolds (called connections of Type $A$ in $[1,8]$ ).
\end{abstract}

2000 Mathematics Subject Classification: Primary 53B05; Secondary 53B20

Keywords: manifold, linear connection, metric connection, pseudo-Riemannian geometry

\section{INTRODUCTION}

In [17], we developed a simple method which helps to decide whether a given linear connection on a 2-dimensional manifold arises as a Riemannian connection of some pseudo-Riemannian metric. Note that in an arbitrary dimension, the problem is difficult to solve. In the real analytic Riemannian case, probably the most effective method was offered by O. Kowalski in [6,7], see also [14-16]. In the simplest case, for nowhere flat two-manifolds, we formulated the necessary and sufficient conditions for local metrizability in [17], and in favourable case, we described all compatible metrics in terms of the Ricci tensor. The purpose of the paper is to show a particular application, related to the class of locally homogeneous connections in 2-manifolds, $[1,8,11]$. First, recall some notation. Let $\left(M_{n}, \nabla\right)$ be a manifold with a linear connection and let $R$ denote the corresponding curvature tensor, $R(X, Y) Z=$ $\left[\nabla_{X}, \nabla_{Y}\right] Z-\nabla_{[X, Y]} Z=\nabla_{X}\left(\nabla_{Y} Z\right)-\nabla_{Y}\left(\nabla_{X} Z\right)-\nabla_{[X, Y]} Z$ for $X, Y, Z$ from $\mathcal{X}(M)$. The induced map $Z \mapsto R(X, Y) Z, R(X, Y): T_{x} M \rightarrow T_{x} M$ for $X, Y, Z$ from $T_{x} M$ is linear and skew-symmetric. The Ricci tensor Ric in type $(0,2)$ is a

The author was supported by the grant from Grant Agency of Czech Republic No. P201/11/0356, Grant No. PrF 2013 022, and by the project of specific university research No. FAST-S-13-2088 of the Brno University of Technology. 
trace of the endomorphism: $\operatorname{Ric}(Y, Z)=\operatorname{Tr}\{X \mapsto R(X, Y) Z\}, X, Y, Z \in X(M)$, hence it carries less information than $R$ in general. The formula

$$
\operatorname{Ric}(Z, Y)-\operatorname{Ric}(Y, Z)=\operatorname{Tr} R(Y, Z)
$$

holds [10, p. 14].

\subsection{The Ricci tensor of a pseudo-Riemannian manifold}

On a pseudo-Riemannian manifold $\left(M_{n}, g\right)$ with the metric tensor $g$, besides the curvature $R$ in type $(1,3)$, we consider the type $(0,4)$ tensor introduced, up to a sign, as $\widetilde{R}(X, Y, Z, W)=g(R(X, Y) Z, W)$, usually also called the curvature tensor; the relations

$$
\widetilde{R}(X, Y, Z, W)=\widetilde{R}(Z, W, X, Y)=-\widetilde{R}(Y, X, Z, W)=-\tilde{R}(X, Y, W, Z)
$$

hold. In a coordinate system $\left(U,\left(x^{i}\right)\right)$ based at a point $x \in M$, components $R_{h i j k}$ of $\tilde{R}$ and $R_{i j k}^{\ell}$ of $R$ satisfy $R_{h i j k}=R_{j k h i}=-R_{i h j k}=-R_{h i k j}, R_{h i j k}=g_{h s} R_{i j k}^{s}$, and $g^{\ell h} R_{h i j k}=R_{i j k}^{\ell}$. On $\left(M_{n}, g\right)$, the Ricci tensor of type $(1,1)$ has components $R_{j}^{i}=g_{s}^{i} R_{s j}$, and the scalar curvature $\varrho$ is its trace,

$$
\varrho=\operatorname{Tr} \operatorname{Ric}=R_{s}^{s}=g^{i j} R_{i j} .
$$

Recall that a Riemannian manifold $\left(M_{n}, g\right)$ is an Einstein space if there is a real constant $\lambda$ such that $\operatorname{Ric}(X, Y)=\lambda g(X, Y) .\left(M_{n}, g\right)$ is isotropic in a point $x \in$ $M$ if the curvature is the same constant, $K(x)$, on every (two-plane) section, and isotropic if it is isotropic in every point, [3]. If $x$ is an isotropic point of $\left(M_{n}, g\right)$, then the following formula holds in $x$ in any local coordinates around $x: R_{h i j k}=$ $K\left(g_{h j} g_{i k}-g_{h i} g_{j k}\right)$.

From now on, suppose $n=2$. Let $\left(x^{1}, x^{2}\right)$ denote local coordinates in a coordinate neighborhood $U$ of a manifold $\left(M_{2}, g\right)$. In dimension two, the non-zero components of $\widetilde{R}$ are equal $R_{1212}$ up to a sign, and the sectional curvature

$$
K(x)=\frac{R_{1212}}{g_{11} g_{22}-g_{12} g_{21}}
$$

is sometimes called the Gaussian curvature ([12, p. 62,], [4, p. 137]). Any twodimensional manifold is isotropic and the formula $R_{h i j k}=K\left(g_{h j} g_{i k}-g_{h i} g_{j k}\right)$ holds [4, p. 137]. Components of the Riemann curvature $R$ of type $(1,3)$ are related to the Ricci tensor by $R_{h j k}^{i}=\delta_{j}^{i} R_{k h}-\delta_{k}^{i} R_{j h},[9,12]$. We easily check by direct evaluation (cf. $[12,17])$ :

Lemma 1. The curvature tensor of a pseudo-Riemannian two-manifold $\left(M_{2}, g\right)$ satisfies

$$
R_{h j k}^{i}=K(x)\left(\delta_{k}^{i} g_{h j}-\delta_{j}^{i} g_{h k}\right)
$$


where $K(x)$ is the Gaussian curvature and the Ricci tensor is a multiple of the metric tensor:

$$
\text { Ric }=K g=\frac{1}{2} \varrho g, \quad g=\frac{2}{\varrho} \text { Ric }
$$

where $\varrho(x)$ is the scalar curvature.

Consequently, the Ricci tensor of the Levi-Civita connection of a 2-dimensional pseudo-Riemannian manifold $\left(M_{2}, g\right)$ is always symmetric and is proportional to the metric tensor. As a corollary, the Ricci tensor is non-degenerate for a nowhere flat $\left(M_{2}, g\right)$.

Obviously, $\left(M_{2}, g\right)$ is an Einstein space if and only if it has constant (scalar or sectional) curvature (cf. [2, p. 44]). If this is the case, then (due to $\nabla g=0$ ) the Ricci tensor is covariantly constant, $\nabla$ Ric $=0$, and vice versa.

Lemma 2. ([17]) The Ricci tensor of a nowhere flat 2-dimensional pseudo-Riemannian manifold $\left(M_{2}, g\right)$ with non-zero sectional curvature is recurrent, i.e.

$$
\nabla \text { Ric }=\omega \otimes \text { Ric, }
$$

and the corresponding (non-zero) 1-form is exact, $\omega=d f$ for some function $f$.

Cf. [5, I, p. 280].

\subsection{Two-manifolds with a linear connection}

Let us now pay attention to a torsion-free linear connection $\nabla$ on a 2-manifold $M_{2}$. As is well known, in this case, the curvature tensor $R$ of type $(1,3)$ can be completly recovered from the Ricci tensor,

$$
R(X, Y) Z=\operatorname{Ric}(Y, Z) X-\operatorname{Ric}(X, Z) Y
$$

for $X, Y, Z \in \mathcal{X}(M)$. In components $R_{h j k}^{i}=\partial_{j}^{i} R_{h k}-\partial_{k}^{i} R_{j h} ; R_{h i j}^{i}=R_{j h}$ for $j \neq i$ and $R_{h j j}^{i}=0$. We get immediately

Lemma 3. In $\left(M_{2}, \nabla\right), R=0$ if and only if Ric $=0$.

Lemma 4. For $\left(M_{2}, \nabla\right)$, the Ricci tensor Ric is recurrent if and only if $R$ is recurrent.

Proof. If Ric is recurrent, then there is a one-form $\omega=\omega_{j} d x^{j}$ such that in local coordinates,

$$
\nabla_{\ell} R_{h j k}^{i}=\delta_{j}^{i} \nabla_{\ell} R_{k h}-\delta_{k}^{i} \nabla_{\ell} R_{j h}=\delta_{j}^{i} \omega_{\ell} R_{k h}-\delta_{k}^{i} \omega_{\ell} R_{j h}=\omega_{\ell} R_{h j k}^{i}
$$

and, hence, $\nabla R=\omega \otimes R$. The converse is similar: if $R$ is recurrent, then $\nabla_{\ell} R_{j k}=$ $\omega_{\ell} R_{k i j}^{i}=\omega_{\ell} R_{j k}$, and $\nabla$ Ric $=\omega \otimes$ Ric.

Since, in the case $R=0$, the tensor Ric is in fact also recurrent $(\omega=0)$, recurrency is a necessary condition for metrizability of two-manifolds. 


\section{MetrizABILITY OF 2-MANIFOLDS WITH LINEAR CONNECTION}

As far as $R_{h j j}^{i}=0$ and $R_{h i j}^{i}=R_{j h}$ hold for $j \neq i$, the curvature tensor of a linear connection $\nabla$ on $M_{2}$ is completely determined by its Ricci tensor; explicitly,

$$
\begin{aligned}
& R_{11}=-R_{112}^{2}=R_{121}^{2}, \\
& R_{21}=-R_{121}^{1}=R_{112}^{1}, \\
& R_{12}=-R_{212}^{2}=R_{221}^{2}, \\
& R_{22}=-R_{221}^{1}=R_{212}^{1} .
\end{aligned}
$$

It is now clear that there are two necessary conditions for local metrizability of a symmetric connection on a pseudo-Riemannian 2-manifold: the Ricci tensor must be symmetric and must also be recurrent for some closed 1-form (Lemma 2). Moreover, Ric may be degenerate only if $R=0$ holds, and if this is the case, then Ric $=0$. For the sake of global metrizability, the corresponding 1-form must also be exact. As is well known, a flat connection is always globally metrizable; we can even prescribe the signature.

When the Ricci tensor (or equivalently, the curvature) is non-zero in one fixed point $x_{0} \in M$, then due to continuity, it is non-zero in some neighborhood of $x_{0}$. Note that the subset of non-flat points is open. The following can be proved:

Theorem 1. Let $\left(M_{2}, \nabla\right)$ be a 2-manifold with a torsion-free linear connection such that the Ricci tensor is symmetric, $R_{i j}=R_{j i}$, recurrent, i.e. (1.1) holds for some 1-form $\omega$, and the regularity condition $\left|R_{i j}\right| \neq 0$ is satisfied. Then, at least locally, there is a metric compatible with the connection.

Theorem 2. Let $\left(M_{2}, \nabla\right)$ be a two-dimensional manifold with a complete torsionfree linear connection and with the curvature $R$ non-zero everywhere. If the Ricci tensor of $\nabla$ is regular, symmetric, and (1.1) holds were the 1-form is gradient, i.e., $\omega=d f$ for some function $f$, then $g=e^{-f}$. Ric is a global metric tensor such that $\nabla g=0$.

Hence, a now-where flat complete torsion-free linear connection on $M_{2}$ is metrizable if and only if its Ricci tensor is symmetric, regular and recurrent with the corresponding 1-form being exact. If this is the case and $\nabla$ Ric $=d f \otimes R i c$ holds for some smooth function $f$, then there is a family of (mutually homothetic) global metrics corresponding to $\nabla, g_{t}=\exp (-f+t) \cdot \operatorname{Ric}, t \in \mathbb{R}$.

The cases

(i) Ric $=0$ identically

and

(ii) Ric is symmetric, recurrent and non-degenerate 
are always favourable for local metrizability of a 2-manifold. Isolated flat points do not matter. But, as examples show, the Ricci tensor might vanish in some "greater" subset and then we can hardly give any reasonable prediction.

\section{TORSION-FREE CONNECTIONS WITH CONSTANT CHRISTOFFELS IN DIMENSION 2}

Consider $n=2$. Let us apply the above methods, cf. [17], in the case of torsionfree connections with constant Christoffels on 2-manifolds (motivation for examining this class comes from classification of locally homogeneous linear connections, [1, 8,11]). A torsion-free connection $\nabla$ in a domain $U \subset M_{2}$ is given uniquely by a family of functions (components) $A(u, v), \ldots, F(u, v)$ in $u, v$ :

$$
\nabla_{\partial u} \partial u=A \partial u+B \partial v, \quad \nabla_{\partial u} \partial v=C \partial u+D \partial v, \quad \nabla_{\partial v} \partial v=E \partial u+F \partial v,
$$

where $\partial u=\frac{\partial}{\partial u}$, similarly for $v$, and $(u, v)$ are local coordinates in $U$ (i. e. $\Gamma_{11}^{1}=A$, $\Gamma_{11}^{2}=B$ etc.). In the case of constant Christoffels, the curvature and the Ricci tensor are constant,

$$
\begin{aligned}
& R_{11}=B(F-C)+D(A-D), \\
& R_{12}=R_{21}=C D-B E, \\
& R_{22}=E(A-D)+C(F-C),
\end{aligned}
$$

and constant components of the covariant derivative $\nabla$ Ric are

$$
\begin{aligned}
& -R_{11 ; 1}=2\left(A R_{11}+B R_{12}\right), \\
& -R_{11 ; 2}=2\left(C R_{11}+D R_{12}\right), \\
& -R_{12 ; 1}=C R_{11}+(A+D) R_{12}+B R_{22}, \\
& -R_{12 ; 2}=E R_{11}+(C+F) R_{12}+D R_{22}, \\
& -R_{22 ; 1}=2\left(C R_{12}+D R_{22}\right), \\
& -R_{22 ; 2}=2\left(E R_{12}+F R_{22}\right) .
\end{aligned}
$$

Lemma 5. A torsion-free linear connection on $M_{2}=\mathbb{R}^{2}[u, v]$ with constant Christoffels is locally flat in the following cases:

(a) $B=D=0, A, C, E, F \in \mathbb{R}$ are related by $A E-C^{2}+C F=0$;

(b) $D \neq 0, C=B E / D, A, B, E, F \in \mathbb{R}$ are related by $D^{2} A-E B^{2}+D B F-$ $D^{3}=0$.

Proof. Suppose Ric $=0$ holds. From technical reasons, let us distinguish the cases $D=0, D \neq 0$ in our considerations (although $D$ is not a preferable coefficient, we can start with something else, e.g. with $C$; the main purpose is only to prepare the situation for application of Maple).

Let $D=0$. Then, the condition $R_{12}=0$ implies $B E=0$. If $E=0$ as well, then we get $B(F-C)=0=C(F-C)$ from (3.1) and further either $B=C=0$ holds, we get the subcase 
(a1) $B=C=D=E=0, A, F \in \mathbb{R}$, or $F=C$, we obtain the subcase

(a2) $D=E=0, F=C, A, B, C \in \mathbb{R}$;

the condition from (a) obviously holds. If $D=B=0$, then the equation $C(F-$ $C)+A E=0$ must hold, case (a).

Now let $D \neq 0$. We get $C=B E / D$ from $R_{12}=0$. The other two components of the Ricci tensor vanish if and only if the following holds:

$$
\begin{aligned}
B(F-B E / D)+D(A-D) & =0, \\
(B E / D)(F-B E / D)+E(A-D) & =0 .
\end{aligned}
$$

This system is equivalent to the unique third-order algebraic equation $D^{2} A-$ $E B^{2}+D B F-D^{3}=0$, hence (b) is checked.

Note that if we choose $D \neq 0$ and either $A, B, E$ or $B, E, F$, we calculate the remaining coefficients uniquely. It means:

Corollary 1. The class of torsion-free linear connections with constant Christoffels contains a three-parameter family of metrizable locally flat connections.

Step by step, the following can be proved, [13].

Lemma 6. If the Ricci tensor of a torsion-free linear connection is recurrent on $M_{2}=\mathbb{R}^{2}[u, v]$, has constant Christoffels and satisfies $R_{12}=0$ then either Ric vanishes or is degenerate.

Lemma 7. If the Ricci tensor a torsion-free linear connection with constant Christoffels on $M_{2}=\mathbb{R}^{2}[u, v]$ is symmetric, recurrent, non-vanishing and non-degenerate then the components must satisfy $R_{11} \cdot R_{22} \neq 0$.

Hence, if a torsion-free connection with constant Christoffels on $M_{2}$ and nonvanishing Ricci tensor should be symmetric and recurrent, then it satisfies necessarily $R_{11} \cdot R_{12} \cdot R_{22} \neq 0$.

Lemma 8. If $\left(M_{2}, \nabla\right)$ is a 2-manifold with a torsion-free linear connection which has constant Christoffels, the corresponding Ricci tensor is recurrent and all its components are non-vanishing on $M_{2}$, then Ric is degenerate.

Corollary 2. In the above notation, exactly the following choices of constants give rise to a metrizable connection $\nabla$ on $M_{2}$ :

(1) $B=C=D=E=0, A, F \in \mathbb{R}$;

(2) $D=E=0, F=C, A, B, C \in \mathbb{R}$;

(3) $B=D=0, A, C, E, F \in \mathbb{R}$ satisfying the equality $A E-C^{2}+C F=0$;

(4) $D \neq 0, C=B E / D, A, B, E, F \in \mathbb{R}$ satisfying the equality $D^{2} A-E B^{2}+$ $D B F-D^{3}=0$. 
Theorem 3. The only (locally) metrizable connections in the class of torsionfree linear connections with constant Christoffels defined on 2-manifolds are exactly locally flat connections.

Note that Theorem 3 follows also from the results published in [1].

\section{REFERENCES}

[1] T. Arias-Marco and O. Kowalski, "Classification of locally homogeneous linear connections with arbitrary torsion on 2-dimensional manifolds," Monatsh. Math., vol. 153, pp. 1-18, 2008.

[2] A. L. Besse, Einstein Manifolds. Berlin: Springer, 1987.

[3] W. M. Boothby, An Introduction to Differentiable Manifolds and Riemannian Geometry, Revised second ed. Amsterdam, London, New York, Oxford, Paris, Tokyo: Academic Press, 2003.

[4] J. Jost, Riemannian Geometry and Geometric Analysis. Berlin, Heidelberg, New York: Springer, 2005.

[5] S. Kobayashi and K. Nomizu, Foundations of Differential Geometry I, II. New York, Chichester, Brisbane, Toronto, Singapore: Wiley-Intersc. Publ., 1991.

[6] O. Kowalski, "On regular curvature structures," Math. Z., vol. 125, pp. 129-138, 1972.

[7] O. Kowalski, "Metrizability of linear connections on analytic manifolds," Note di Matematica, vol. 8, no. 1, pp. 1-11, 1988.

[8] O. Kowalski, B. Opozda, and Z. Vlášek, "A classification of locally homogeneous connections on 2-dimensional manifolds vis group-theoretical approach," CEJM, vol. 2, no. 1, pp. 87-102, 2004.

[9] D. Lovelock and H. Rund, Tensors, Differential Forms, and Variational Principle. New York, London, Sydney: A. Wiley-Intersc. Publ., 1975.

[10] K. Nomizu and T. Sasaki, Affine Differential Geometry. Geometry of Affine Immersions. Cambridge Univ. Press, 1994.

[11] B. Opozda, "A classification of locally homogeneous connections on 2-dimensional manifolds," Diff. Geom. Appl., vol. 21, pp. 173-198, 2004.

[12] A. Z. Petrov, Einstein Spaces. Oxford: Pergamon Press, 1969.

[13] P. Pirklová, "Metrizability of linear connections," Ph.D. Thesis, Palacký University Olomouc, Olomouc, 2012, (Metrizovatelnost afinní konexe, in Czech).

[14] A. Vanžurová, "Metrization of linear connections, holonomy groups and holonomy algebras," Acta Physica Debrecina, vol. 42, pp. 39-48, 2008.

[15] A. Vanžurová, "Metrization problem for linear connections and holonomy algebras," Archivum Mathematicum (Brno), vol. 44, pp. 339-348, 2008.

[16] A. Vanžurová, "Metrization of connections with regular curvature," Archivum Mathematicum (Brno), vol. 45, no. 4, pp. 325-333, 2009.

[17] A. Vanžurová and P. Žáčková, "Metrizability of connections on two-manifolds," ACTA UPO, Math., vol. 48, pp. 157-170, 2009.

Author's address

Alena Vanžurová

Palacký University, Faculty of Science, Department of Algebra and Geometry, Tř. 17. listopadu 12, 77146 Olomouc, Czech Republic

Current address: Brno University of Technology, Faculty of Civil Engineering, Department of Mathematics, Veveř́ 331/95, 60200 Brno, Czech Republic

E-mail address: alena.vanzurova@upol.cz and vanzurova.a@fce.vutbr.cz 